\title{
No Evidence for Effects of Fitness Relevance or Sex Differences in a Virtual Hunting and Gathering Task
}

\author{
Michael F. Brown ${ }^{1} \cdot$ Jared M. Batterman $^{1} \cdot$ Joseph Leman $^{1} \cdot$ Bethany Taylor $^{1}$. \\ Jung Kim ${ }^{1}$ - Jennie Ruff ${ }^{1}$ • Marie E. Saxon ${ }^{1}$ - Andrew Stokesbury ${ }^{1}$
}

Published online: 4 December 2015

(C) Springer International Publishing 2015

\begin{abstract}
A computerized task could be completed by predicting the location of a moving target or by choosing several stationary targets. In a fitness-relevant condition, this task was presented to participants in terms of hunting and gathering food necessary for survival. In four experiments, there was no evidence that male and female participants differed in terms of their tendency to complete the task in these two ways. In three of the experiments, performance in the fitness-relevant condition was compared to performance in a control condition in which the task was presented as a computer game with no reference to hunting or gathering. No evidence for an effect of fitness relevance was found. These results challenge the idea that sex differences in spatial cognition are related to the sexual differentiation of foraging behavior during human history. They also suggest limitations on the role of fitness-relevant processing of information ("survival processing") on cognitive performance.
\end{abstract}

Keywords Spatial cognition - Sex differences $\cdot$ Survival processing $\cdot$ Gathering hypothesis

It has been argued that human spatial cognition was shaped by our evolutionary history as hunter-gatherers. A particularly influential version of this idea was proposed by Silverman and Eals (1992), who concluded that qualitatively different spatial abilities resulted from the sexual differentiation of gathering and hunting activities in human females and males.

Michael F. Brown

michael.brown@villanova.edu

1 Villanova University, Villanova, PA, USA
Silverman and Eals (1992; Eals and Silverman 1994) described a foraging adaptation hypothesis, according to which differences in male and female spatial performance exist because of the ancestral roles of men as hunters and women as gatherers. They argued that male performance advantages that had been shown in many spatial tasks (see Halpern 2000; Linn and Petersen 1985; Voyer et al. 2007; Voyer et al. 1995 for reviews) exist because most of the tasks that had been used were structured in a way that emphasized spatial skills and processes related to hunting. They hypothesized that spatial tasks more relevant to gathering would support more efficient or accurate performance by females (see also Postma et al. 1998). To test this, Silverman and Eals used arrays of images of common objects. Participants were asked to examine the array during a study period, after which it was removed. They were then presented with a second array containing all of the same objects as the first, in the same locations, but with additional objects added. Participants were then required to identify objects that were not in the original array. Following this, participants were given yet another array; this one comprised of all the same items as the first display, but with some of the items in different locations. They were asked to identify the objects that had and had not been moved. There was a significant female advantage in both of these object location memory tasks. Silverman and Eals argued that object location memory was a component of the skills supporting gathering and that the sex difference in performance is best attributed to the sexual differentiation of foraging behaviors in human history.

Silverman and Eals's basic result has been replicated a number of times (e.g., Choi and Silverman 1996; Dabbs et al. 1998; McBurney et al. 1997), including a crosscultural study involving a large, worldwide sample of participants (Silverman et al. 2007). Their conclusions continue to be very influential, but they have also generated debate on 
several fronts. In some experiments, the female superiority of object location memory is not found or found only under a limited set of conditions (e.g., Honda and Nihei 2009; Iachini et al. 2005; James and Kimura 1997; Postma et al. 2004). Cashdan et al. (2012) report the absence of female superiority of object location memory in Hazda hunter-gathers (see also Vashro and Cashdan 2015). Thus, there is ambiguity about the scope of the basic result.

A related area of controversy is the extent to which the performance differences found in these experiments are best attributed to sex differences in spatial memory as opposed to other cognitive domains. In particular, several groups of investigators have argued that the female advantage in object location memory is based on sex differences in verbal memory rather than spatial cognition (Barnfield 1999; Choi and L'Hirondelle 2005; James and Kimura 1997).

It has also been pointed out that sex differences in spatial performance are widely reported in non-human mammals that do not have an evolutionary history of sexual differentiation of hunting and gathering tasks like that of humans (Saucier et al. 2007). An alternative explanation of sex differences in spatial cognition, proposed by Gaulin and FitzGerald (1986), is the polygyny-range size hypothesis (see also Ecuyer-Dab and Robert 2004; Gaulin 2006; Gaulin and Hoffman 1998; Leibowitz and Brown 1999; Saucier et al. 2007). In its initial form, this hypothesis dealt mainly with male spatial superiority in spatial performance, explaining it as an evolutionary adaption in polygynous species serving males ability to seek out viable mates across large areas of land. However, more recent forms of the idea also attempt to explain female advantages in object location memory (Ecuyer-Dab and Robert 2004). While the polygyny-range size hypothesis is not incompatible with Silverman and Eals' theory (different species often evolve similar mechanisms for entirely different reasons or for multiple reasons), it does offer a viable alternative.

Another domain in which evolutionary influences on cognitive processes have been explicitly explored is incidental memory for verbal material. Nairne et al. (2007) introduced a line of research investigating the effect of evolutionary relevant cognitive processes ("survival processing") on verbal memory. The effect of survival processing was found in an incidental recall test. Participants in their first experiment were given a list of common nouns and told to rate them in terms of importance of the object corresponding to the word for survival in a life-or-death situation, in the context of a household move, or rate the words on a scale of pleasantness. In an unexpected recall test, recall of the words was substantially better in the survival processing condition.

Similarly, Nairne et al. (2009) assigned participants to one of three groups: gathering, hunting, or scavenger hunt. They were then presented with a vignette which told a different story depending on which condition they were assigned. In the gathering condition, participants were told to imagine they were living a long time ago in grasslands and that they were tasked with gathering edible food for their tribe. Regardless of weather conditions, availability, etc., they were told that if they did not gather enough food, their tribe would not survive. The vignette for the hunting scenario was otherwise the same, but replaced gathering with hunting. The scavenger hunt condition asked participants to imagine they were taking part in a team scavenger hunt, and included information about how to acquire these items in both a "hunting" and a "gathering" manner (without actually using those words, something that they did do in the previous vignettes). Participants were told that in order for their team to win, they themselves must scavenge successfully. Following whichever vignette they received, participants were asked to rate 30 objects in terms of how useful it would be for the task of hunting, gathering, or participating in a scavenger hunt. After a distractor task was completed, participants were given an incidental recall test on the object names they rated. The key finding was significantly greater recall in the hunting and gathering conditions than the scavenger hunt control condition. The data were interpreted as demonstrating that incidental memory for verbal material was enhanced when the remembered information was relevant to survival or fitness.

This basic pattern of results - better memory for verbal material processed in a fitness-relevant manner-has been replicated and extended along several lines (e.g., Kang et al. 2008; Nairne and Pandeirada 2008; Nairne and Pandeirada 2010; Nairne et al. 2012; Otgaar et al. 2010; Weinstein et al. 2008; see Nairne 2010 for a review).

The present experiments were designed to explore the roles of fitness-relevant processing and sex in a task intended to emulate some of the contingencies, skills, and processes related to hunting and gathering. The task required participants to choose from among marked locations in a two-dimensional matrix of 36 locations arranged on a computer display. Trials of the task could be completed in two ways. The first was to anticipate the location of a stimulus that moved from choice to choice in a somewhat predictable manner. The moving target was hidden from view most of the time but, following each choice, briefly appeared in a new location. This task was intended to have in common with hunting that a moving target had to be tracked and its location predicted based on imperfect information. A trial could also be completed by choosing a number of targets, the location of which was fixed during the trial (stationary targets). This was intended to have in common with gathering that several fixed locations must be chosen and (in three of the four experiments) memory was required to locating the targets efficiently (the nature of the memory requirement varied over experiments).

The task was presented to participants in two different formats. In fitness-relevant conditions, the moving target was an image of a prey animal and the stationary (gathering) targets were images of vegetables. The participants in the fitness- 
relevant condition read an instructional vignette intended to frame the task as a food gathering that was critical for survival of the participant's imagined group. The vignette was very similar to the ones used in the experiments of Nairne and colleagues. In particular, there was emphasis on the survival relevance of food procurement. In a control condition, the target stimuli were simple shapes. The vignette framed the task as a computer game without referring to anything related to survival, hunting, or gathering food.

These experiments had three primary goals. The first was to explore the possibility that fitness relevance would enhance the spatial performance measured by this task. Performance on the task in the fitness-relevant (hunting and gathering) condition was compared to performance in the control (video game) condition. The second goal was to determine whether there are sex differences in performance on the task. Of particular interest was the possibility that males would tend to complete the task by locating the moving target whereas females would tend to complete the task by choosing the stationary targets. Finally, the third goal was to determine whether any sex difference in this tendency was modulated by the fitness relevance of the task. That is, would a sex difference in the tendency to track the moving target ("hunt") versus locate the stationary targets ("gather") be greater when the task is framed in the fitness-relevant manner?

\section{Experiment 1}

In the first experiment, there were nine stationary targets and they formed a $3 \times 3$ matrix within the larger matrix of possible locations on the display. The location of the stationary targets varied unpredictably over trials. The location of the stationary targets was revealed briefly (for $2 \mathrm{~s}$ ) following choice of the first of them. Thus, after choosing the first stationary target, participants could remember the location of the remaining stationary targets. The moving target changed locations following each choice in a manner that could not be predicted exactly but was consistent in terms of the direction of movement and the approximate distance moved from choice to choice. This was intended to emulate the somewhat predictable movement of prey when hunting. The performance of female and male participants in a fitness-relevant condition and control condition was compared. The key questions were as follows: (1) Would performance be improved by fitnessrelevant processing? (2) Would there be a sex difference in the tendency to attempt to locate the moving target vs. remember and choose the locations of the stationary targets? (3) Would a sex difference in the tendency to locate the moving target vs. remember and choose the stationary targets be dependent on (or modulated by) fitness-relevant processing?

\section{Method}

Participants Eighty-four Villanova University students (42 female and 42 male) participated as part of a research requirement in an introductory psychology course or to receive extracredit in a higher level course.

Materials and Apparatus Stimuli were displayed on standard LCD computer monitors. Participants were required to make choices by utilizing a computer mouse and moving the on-screen cursor over the chosen area of the experimental matrix and clicking the mouse button. All stimuli and procedures (with exception of the instructions and the debriefing) were implemented utilizing a program created with Microsoft Visual Basic v. 6.0.

The experiment was conducted in a classroom with approximately 25 computer stations present. Eight of these computer stations were configured to conduct the present experiment. They were maximally distanced from each other within this space. Up to six participants were tested simultaneously, although two or three at one time was the most common.

Procedure Before their arrival at the laboratory, each participant was randomly assigned to one of two conditions (fitness relevant or non-fitness relevant), with the constraint that there be an approximately equal number of females and males in each condition. Upon their arrival, they were asked to fill out a standard experimental consent form. Following this, they were seated in front of a computer and asked to read one of two instructional sets (one for each of the two conditions), which were modified versions of the instructional sets used by Nairne et al. (2009). Those in the fitness-relevant condition saw the following:

In this task we would like you to imagine that you are living long ago in the grasslands of a foreign land. As a part of a small group, you are in charge of contributing food for your tribe. You could either hunt big game, or gather edible fruits, nuts, or vegetables. Hunters often have to travel great distances in order to find food, and once they've found it, they often have to track it before they are able to capture it. Gatherers often have to have knowledge about the locations and seasonal availability of edible foods, and to gather most efficiently, they should avoid revisiting areas from which they recently gathered. When you have finished reading these instructions, you will be presented with a matrix of grassland locations. To begin securing food, simply click any of the locations, following which you will see an animal appear for a short time before once again disappearing. The location in which the animal appeared was its location at the time you clicked. To catch it, thus securing food for your tribe, you will need to click the location in 
which you anticipate the animal will be next. If you click in a gathering area (a fertile patch of land, containing fruits, nuts, and/or vegetables), all possible gathering locations will appear for a short time before once again disappearing. To gather enough food for your tribe, you will need to click locations inside the gathering area until the trip ends, signaling that you have gathered enough food for your tribe in this particular area. You will be sent out on 40 expeditions to secure food, each of which can be completed by hunting or gathering. Either strategy can allow you to get enough food to feed your tribe. However, time is of the essence, and it is your goal to finish each expedition as quickly and as accurately as you can. This will also allow you to complete the experiment more quickly.

While those in the control condition saw the instructions below instead:

In this task we would like you to complete a series of computer games. Your job is to finish them as quickly and as accurately as possible. Each game can be completed either by clicking a single moving shape, or by clicking all tiles which contain a hidden stationary shape. When you have finished reading these instructions, you will be presented with a matrix depicting a series of black tiles. To begin a game, simply click any of the tiles, following which you will see the mobile shape appear for a short time before once again disappearing. The location in which it appeared was its location at the time you clicked. To catch it, you will need to click the tile that you anticipate it will be next. If you click where stationary shapes could be hidden, all of their possible locations will appear for a short time before once again disappearing. To get them all, you will need to click all of their possible locations until the trial ends, signaling that you have found all that were hidden in this particular area. You will complete 40 games, each of which can be completed by catching the single moving shape or by finding all of the hidden stationary shapes. Either strategy will allow you to complete the games, thus bringing you one step closer to the end of the experiment. However, time is of the essence, and it is your goal to find the shapes as quickly and as accurately as you can.

After each participant finished reading the instructions, the experimenter turned on the computer monitor, which displayed a $6 \times 6$ matrix of square tiles, each depicting either an identical picture of a grassland landscape (grass field with cloudy sky above the horizon; fitness-relevant condition) or an identical plain monochromatic image (control condition; See Fig. 1). Each tile was $1.1 \mathrm{~cm}$ on each side, with a distance of $1.8 \mathrm{~cm}$ separating individual tiles.

Participants made choices by moving the mouse cursor and clicking the left mouse button within the boundaries of a tile. Each trial ended when one of two conditions was reached: (1) the tile in which the moving target appeared was chosen or (2) all nine tiles designated stationary targets had been chosen. The first choice made during a trial was made with no information about the location of either the moving or stationary targets, as no targets were shown until a choice was made.

When a choice of any tile in the matrix was made, the moving target appeared in a pre-determined location for $3 \mathrm{~s}$ (Fig. 2). During that same 3-s interval, mouse clicks were ineffectual and the mouse cursor changed from the standard MS-Windows ${ }^{\mathrm{TM}}$ mouse cursor arrow to the universal negation icon (circle with diagonal hash). This was done to increase the control of choices by the experimental contingencies by discouraging haphazard choices. The initial location of the moving target (i.e., where it appeared following the first participant choice) was determined randomly from among the 36 locations in the matrix. Following the first choice, the moving
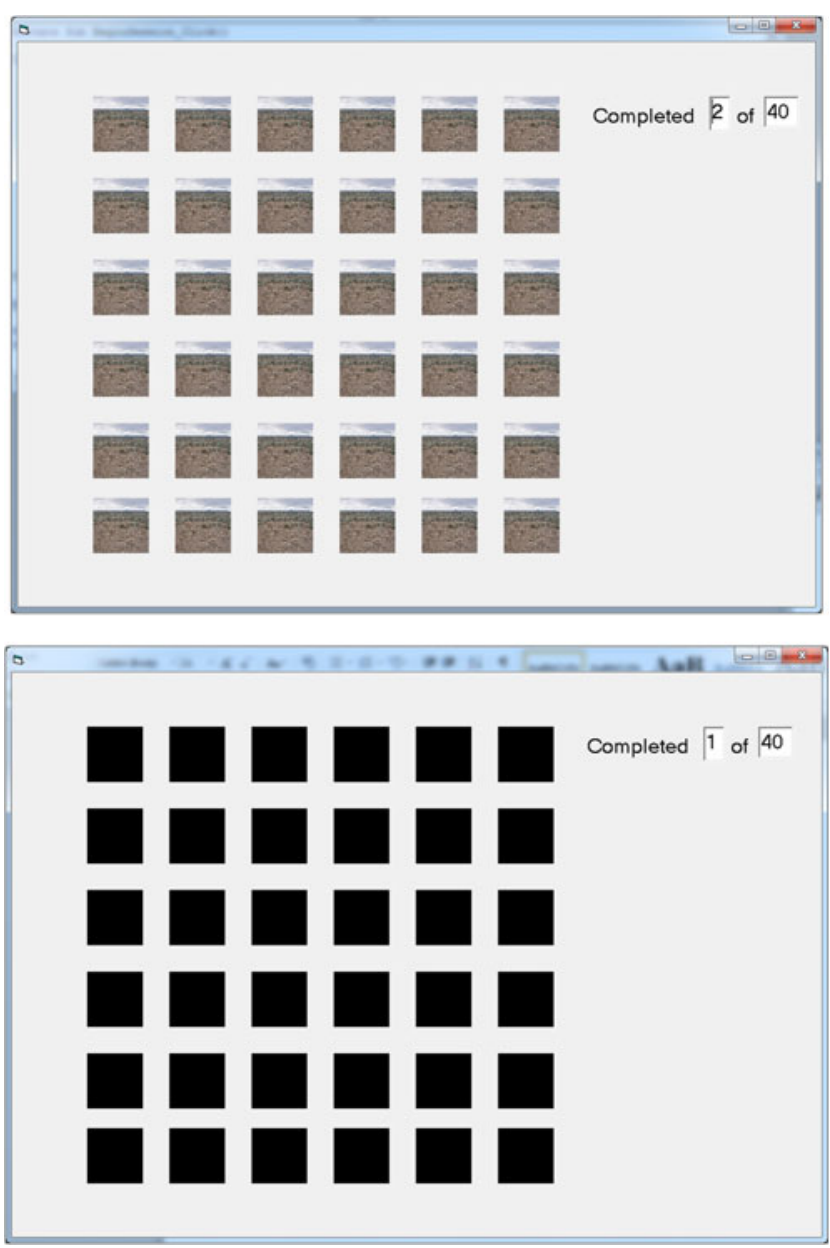

Fig. 1 The matrix of tiles with background stimuli in the fitness-relevant condition (top panel) and control condition (bottom panel) 

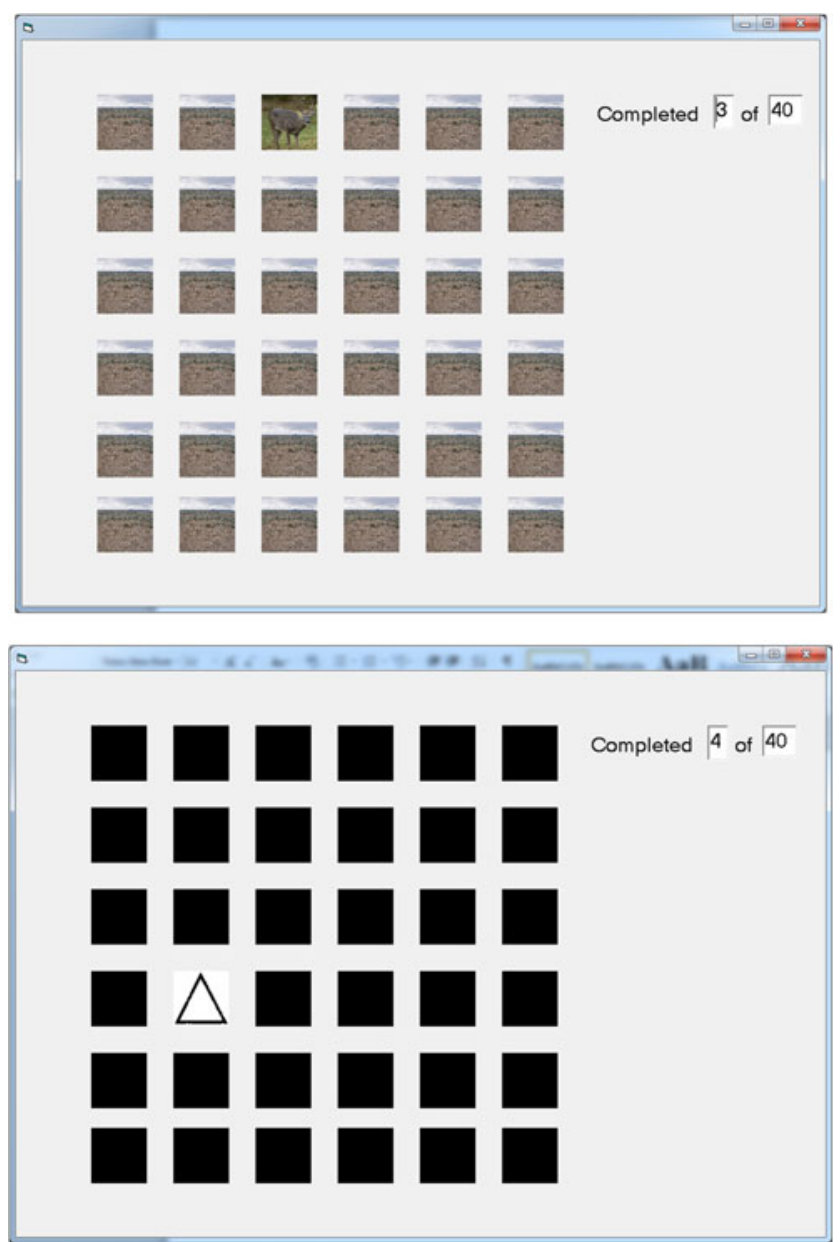

Fig. 2 Examples of a moving target in the fitness-relevant condition (top panel) and control condition (bottom panel)

target moved systematically from choice to choice, according to an algorithm. Following each choice made by the participant, it moved either 1 or 2 tiles horizontally and 1 or 2 tiles vertically from its previous location (the distance moved horizontally was independent of the distance moved vertically). The initial direction of movement in each direction was determined randomly. Following the first choice, the moving target continued in the same two directions (up or down and left or right) until it hit an edge, at which point it reversed itself. For example, if it was moving up and to the right, it would continue to do so until it either hit the right border of the matrix (at which point it would begin moving left), hit the top of the matrix (at which point it would begin moving down), or hit the top right corner of the matrix (at which point both directions would reverse). If the participant clicked the location in which the moving target was scheduled to appear next, the target appeared there for $3 \mathrm{~s}$ and then the trial ended.

On each trial, the stationary targets were nine tiles configured in a 3-tile (wide) $\times 3$-tile (tall) array. The location of this array in the $6 \times 6$ matrix of tiles was randomly determined for each trial (i.e., it was randomly selected from among the 16 possibilities). At the beginning of each trial, and during most of the trial, the stationary targets were comprised of the same background stimuli as non-target tiles. The stimuli revealing the stationary targets were presented at most once during the trial, if and when the participant made her or his first choice of a stationary target. Immediately following that choice of the first stationary target, each of the nine stationary targets were revealed by replacement of the background stimulus with an image of vegetables (fitness-relevant condition) or a shape drawing (control condition; Fig. 3) for $3 \mathrm{~s}$. After $3 \mathrm{~s}$, the stationary targets reverted back to the background image. Thus, the locations of the stationary targets was unknown until one of them was chosen and had to be remembered thereafter. If the participant chose all nine stationary targets, the trial ended.

Three animal images were used as the stimulus appearing as the moving target (a photograph of a single deer, moose, or buffalo; one assigned randomly to each participant in the fitness-relevant condition) and three shapes were used as
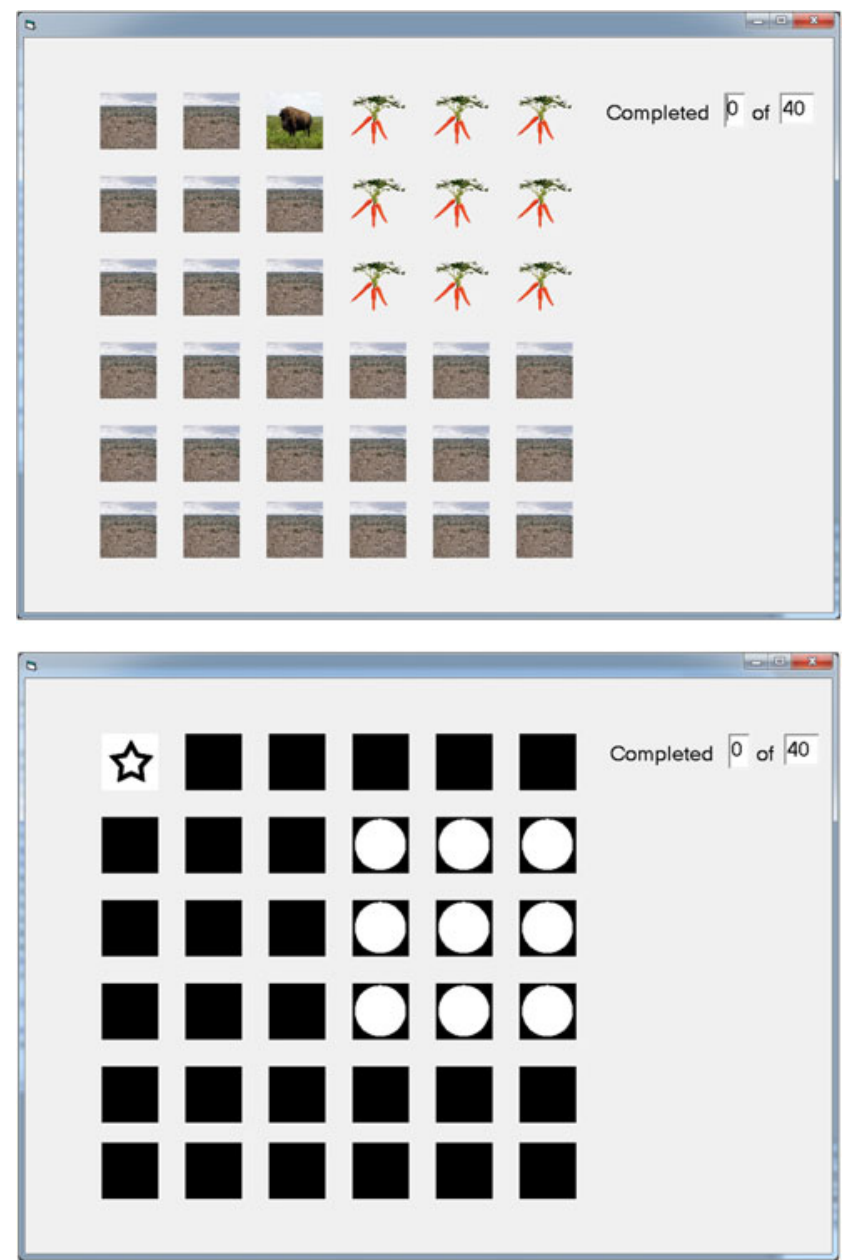

Fig. 3 Examples of a moving target and the stationary targets in the fitness-relevant condition (top panel) and control condition (bottom panel) of experiment 1 . In experiment 1 , both kinds of targets would be visible only briefly following the first choice of a stationary target 
moving targets (drawing of a single triangle, star, or square; one assigned randomly to each participant in the control condition). These appeared as the tile in place of the background image for $3 \mathrm{~s}$ following each participant choice, following which the tile it was on reverted back to the background image (Fig. 2 shows examples of the matrix immediately following a choice). Three images of vegetables were used as the stimuli appearing in the nine stationary target locations when the participant made the first choice of any one of them (photographs of several peppers, potatoes, or carrots; one assigned randomly to each participant in the fitness-relevant condition) and three shapes were used a stationary targets (circle, trapezoid, or pentagon; one assigned randomly to each participant in the control condition). Figure 3 shows examples of the matrix immediately following the first choice of a stationary target.

Participants initiated each trial by using the mouse to click on a button (labeled "Begin Trial") above the display where the tile matrix would appear. Clicking the button resulting in the tile matrix appearing and the "Begin Trial" button disappearing. Completion of a trial (by choosing the moving target or all nine stationary targets) resulted in the tile matrix disappearing (replaced by solid grey background) and the "Begin Trial" button appearing. The upper left part of the display (above and right of the area occupied by the tile matrix) had a message displaying the number of trials completed ("Completed $X$ of 40 Trials"; $X$ being the number of trials completed; Figs. 1, 2, and 3). This feedback to the participant was present during trials as well as between trials. The session ended when the participant completed 40 trials (or after $25 \mathrm{~min}$ if 40 trials were not completed).

Appendix 1 contains screen-captured video examples of how trials in the experiment appeared.

\section{Results}

All 84 participants completed the experiment within $25 \mathrm{~min}$.

The ability of participants to efficiently perform the task was measured as the number of choices required to complete trials (by either locating the moving target or choosing all of the stationary targets). To detect changes in performance with experience, the first 20 trials and last 20 trials were analyzed separately as two-trial blocks. Figure 4 shows the mean number of choices required to complete a trial as function of participant sex, experimental condition, and trial block. These results were evaluated with a $2(\operatorname{sex}) \times 2$ (condition) $\times 2$ (trial block) ANOVA, with the trial block being a within-subject factor. There is a significant effect of trial block, $F(1,80)=18.322, p=$ 0.001 , and a significant effect of $\operatorname{sex}, F(1,80)=4.997, p=$ 0.028 , but no effect of condition and no significant interactions among these factors. Fewer choices were required to complete trials in block 2 than in block 1. Males completed trials in fewer choices than females.
Trials could be completed either by choice of the moving target ("hunting") or by choosing all of the stationary targets ("gathering"). Note that it is possible for a trial to be completed by choice of a tile that is both the moving target location and the location of the last stationary target to be chosen. These cases are not counted in the calculation of this measure. Figure 5 displays the percentage of trials completed by choice of the moving target, as function of sex, experimental condition, and trial block. An ANOVA parallel to the one described above was used to evaluate these results. There was a significant effect of the trial block $F(1,80)=268.047, p=0.001$, but no other significant effects or interactions. The tendency to complete trails by choice of the moving target was greater in the second trial block than in the first.

To examine the tendency to make choices related to the moving target as opposed to the stationary targets in more detail, we classified each choice made in terms of whether it was consistent with choice of the moving target and/or the stationary targets. A choice was considered to be consistent with choosing stationary targets if it was, in fact, one of them. A choice was considered to be consistent with choice of the moving target if it conformed to the algorithm that determined the next location of the moving target. The first choice was not included in this analysis because the participant had no information about the location of the moving target before the first choice. In the case of the second choice, any chosen location that was one or two rows away from the last location of the moving target and one or row columns away from the last location of the moving target was considered to be consistent with choosing the moving target. For choices made after the second choice, this same criterion applied but the chosen location had to also be in correct $x$ and $y$ direction in the matrix (i.e., the directions in which the target was programmed to move). Every choice (other than the first choice of each trial) was thereby classified as consistent with choosing the moving target, the stationary targets, both, or neither.

Figure 6 shows distributions of choices, classified in this manner. The distributions are shown as a function of sex, experimental condition, and trial block. Distributions were calculated for each participant during each trial block, and the figure shows the mean distribution (over participants within each block). To determine differences in the tendencies of make choices of these different kinds, a 3 (choice type) $\times 2$ (trial block) $\times 3$ (choice type) $\times 2($ sex $) \times 2$ (condition) ANOVA was conducted. There are four types of choices in the distributions, but because every choice fit into one of the four categories, the percentage of choices consistent with neither strategy was excluded from analysis to prevent the analysis from violating the assumptions of ANOVA. There was a significant effect of trial block, $F(1,80)=$ 
Fig. 4 The mean number of choices made to complete trials in experiment 1 , as a function of experimental condition and sex in trial blocks 1 (left panel) and 2 (right panel). Error bars indicate one standard error of the mean (S.E.M.)
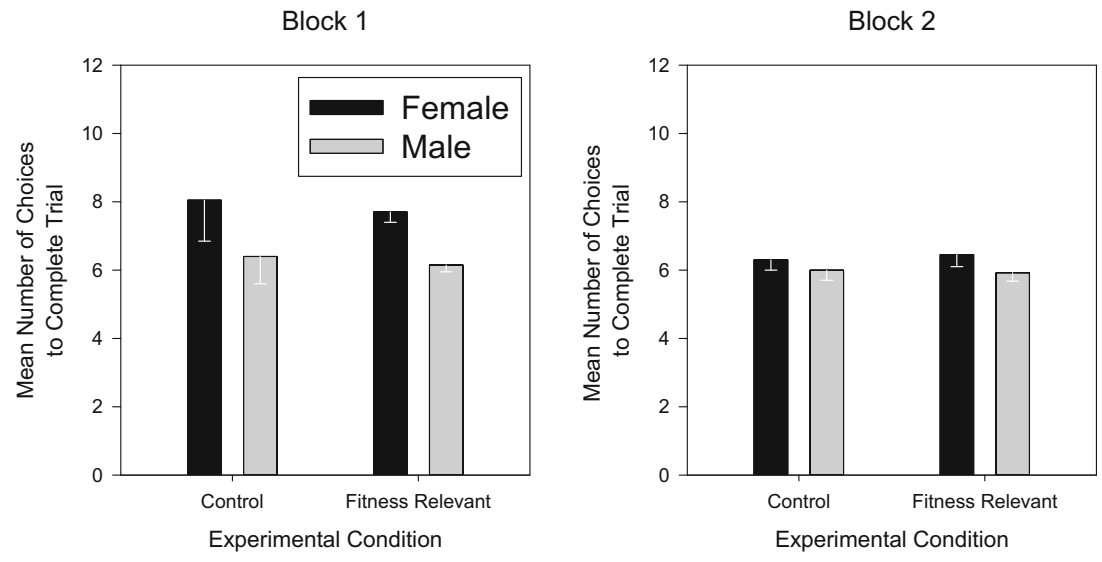

100.982, $p=0.001$ and choice type $F(2,160)=39.253, p=$ 0.001 , and a significant interaction between the two $F(2$, $160)=24.521, p=0.001$. In addition, there was a significant effect of sex, $F(1,80)=5.263, p=0.024$. In order to understand the interaction between trial block and choice type and to further investigate what, if any, effect condition had on individual trial blocks, two 3 (choice type) $\times 2$ $(\operatorname{sex}) \times 2$ (condition) ANOVAs were conducted on the data from block 1 and block 2, respectively. The ANOVA on the block 1 data revealed a significant effect of choice type, $F(2,160)=26.607, p=0.001$, and sex, $F(1,80)=$ 4.604, $p=0.035$. The ANOVA on the block 2 data also revealed effects of choice type, $F(2,160)=44.906, p=$ 0.001 , and sex, $F(1,80)=4.153, p=0.045$. The effects of choice type reflect, in part, more choices consistent with the moving target than with the stationary targets and the trial block $\times$ choice type interaction is due to this difference being greater during block 2 than during block 1 . The effects of sex are due to males making relatively more choices consistent with a target (moving, stationary, or both) than females made. The effect of the trial block is due to there being more choices consistent with targets (moving, stationary, or both) in block 2 than in block 1 .

\section{Discussion}

There was an improvement in choice performance over the two trial blocks, in that fewer choices were required to complete trials during the second trial block than during the first. This appears to be due to increased skill in locating the moving target (hunting), in that more trials were completed by choice of the moving target during the second trial block than during the first and more choices were consistent with the moving target during the second trial block than the first. There was also a sex difference in performance; males required fewer choices than females to complete trials.

Although there was a sex difference in performance, there was no evidence for a sex difference in the relative tendencies to locate the moving target ("hunt") vs. remember and locate the stationary targets ("gather"), as measured by either the kind of choice made to complete a trial or the distribution of choices overall. There was also no evidence for an effect of the fitness relevance manipulation on the number of choices required to complete trials. Like any null result, the lack of evidence for these key differences is relatively difficult to interpret. The remaining experiments are attempts to explore conditions that might reveal effects of the fitness relevance
Fig. 5 The mean percentage of trials completed by choice of the moving target in experiment 1 , as a function of experimental condition and sex in trial blocks 1 (left panel) and 2 (right panel). Error bars indicate one S.E.M.

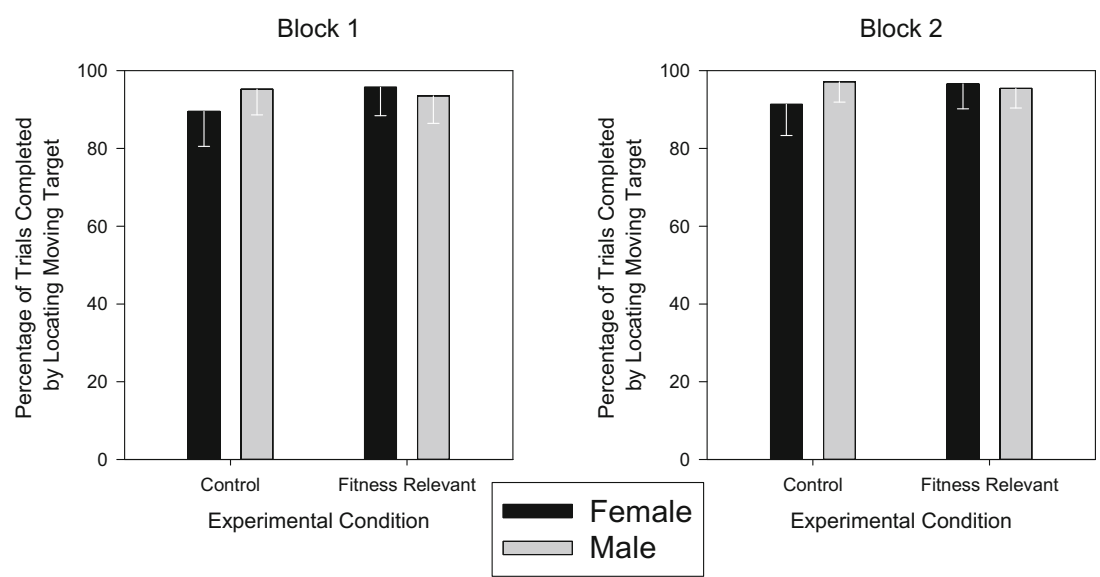


Fig. 6 The mean distributions of choices in experiment 1

consistent with the moving target, of a stationary target, both consistent with the moving target and of a stationary target, and neither consistent with the moving target nor of a stationary target. The first choice of each trial was excluded from these distributions (because no information about the moving target was available at that point) Separate distributions are shown as a function of sex, experimental condition (top vs. bottom panels), and trial block (left vs. right panels) to complete trials in experiment 1 , as a function of experiments condition and sex in trial blocks 1 (left panel) and 2 (right panel). Error bars indicate one S.E.M.
Control Condition - Block 1

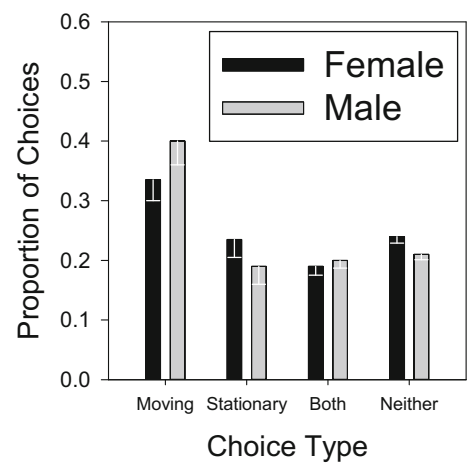

Fitness Relevant Condition - Block 1

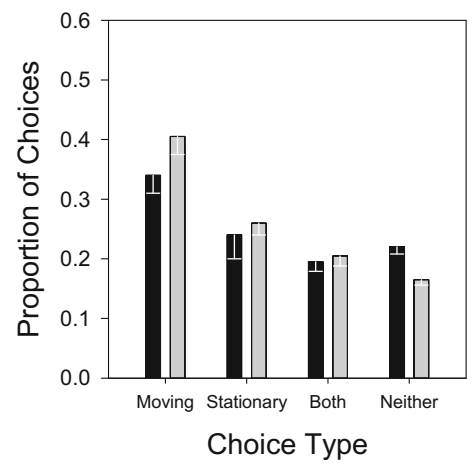

Control Condition - Block 2

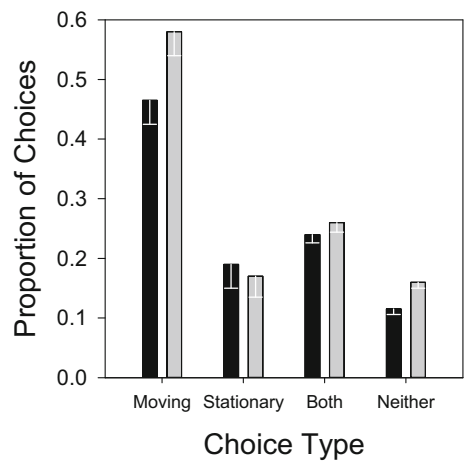

Fitness Relevant Condition - Block 2

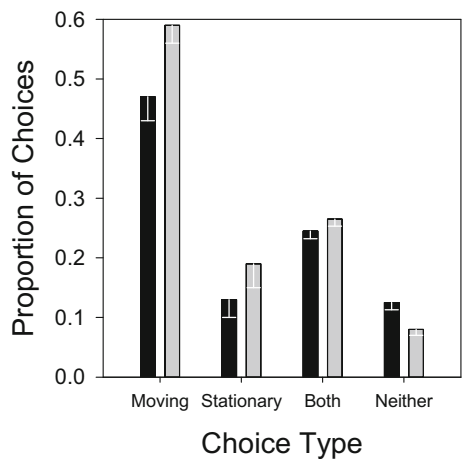

manipulation and a sex difference in tendencies to choose moving vs. stationary targets that were not found in this experiment.

\section{Experiment 2}

A problem with experiment 1 is the large tendency to make choices consistent with the moving target regardless of sex or experimental condition. A task that produced a more balanced tendency to complete the task in the two ways would be more sensitive to differences in the tendency to hunt vs. gather and therefore might better allow effects of these factors to be revealed. Experiment 2 did this by increasing the complexity of the movement of the moving target, thereby making it more difficult to choose the moving target to complete a trial. Specifically, the moving target could move by 0,1 , or 2 rows and by 0,1 , or 2 columns from choice to choice (rather than 1 or 2 rows and 1 or 2 columns in experiment 1). The experimental procedures were otherwise the same as experiment 1 .

\section{Method}

Participants Sixty-four Villanova University students (40 female and 24 male) participated as part of a research requirement in an introductory psychology course.
Materials and Apparatus The materials and apparatus used were the same as in experiment 1 .

Procedure Participants were randomly assigned to two experimental conditions, with constraints intended to result in approximately equal numbers of female and male participants in the fitness-relevant and control conditions. There were 19 and 21 females assigned to the control and fitness-relevant conditions, respectively. The corresponding numbers for males were 14 and 10.

The procedure was identical to that of experiment 1 , with one exception. The moving target moved 0,1 , or 2 rows and 0,1 , or 2 columns from choice to choice. Determination of whether a choice was consistent with the moving target for analysis purposes accommodated this change. Thus, there were more locations in the tile matrix consistent with choice of a moving target and a smaller chance of choosing the correct location among them.

\section{Results}

Preliminary analysis of the data parsed into two trial blocks did not indicate any differences in the pattern of results during the first and second 20 trials. Thus, the data from all 40 trials were combined. The data were otherwise analyzed in the same manner as the data from experiment 1. 
Figure 7 shows the mean number of choices required to complete a trial as function of participant sex and experimental condition. These results were evaluated with a 2 $(\operatorname{sex}) \times 2$ (condition) ANOVA. There is no evidence of an effect of sex, $F(1,60)=2.0$, or condition, $F(1,60)<1$. There was no significant sex $\times$ condition interaction, $F(1,60)<1$.

Figure 8 shows the percentage of trials completed by choice of the moving target, as a function of sex and experimental condition. These results were evaluated with a 2 (sex) $\times 2$ (condition) ANOVA. There is no evidence of an effect of sex, $F(1,60)<1$, or condition, $F(1,60)=1.52$. There was no significant sex $\times$ condition interaction, $F(1$, $60)<1$.

Figure 9 shows distributions of choices, classified according to whether they are consistent with choice of the moving target, stationary target, both, or neither. The distributions are shown as a function of sex and experimental condition. Although choices were classified in the same way they were in experiment 1 , we chose to analyze these results in a different way than those of experiment 1 . Because the focus is on how the tendency to make choices consistent with moving vs. stationary targets varied as a function of sex and experimental condition, we analyzed the results in terms of the number of choices consistent with moving vs. stationary targets (but not both) using an ANOVA. The number of choices was compared with a 2 (choice type: consistent with moving target vs. choice of stationary target) $\times 2(\operatorname{sex}) \times 2$ (condition) ANOVA. There was no main effect of choice type target, $F(1,60)<1$, indicating the tendencies to make choices of the two types did not differ. There were no significant effects involving sex or condition, although the condition $\times$ choice type approached significance, $F(1,60)=2.97, p=$ 0.09 .
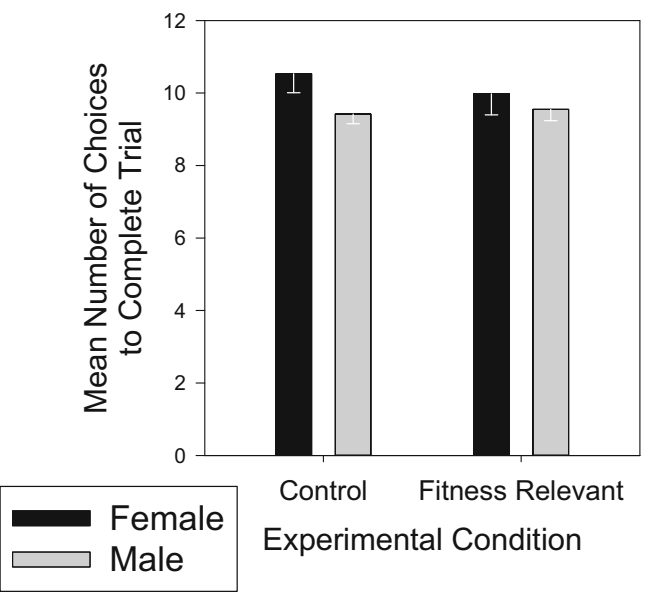

Fig. 7 The mean number of choices made to complete trials in experiment 2, as a function of experimental condition and sex. Error bars indicate one S.E.M.

\section{Discussion}

The change in procedure, relative to experiment 1 , was successful in making the two kinds of choices (and the corresponding two ways of completing trials) about equally likely. Less than $70 \%$ of trails were completed by locating the moving target rather choosing all the stationary targets, rather than over $90 \%$ in experiment 1 . Thus, the procedure of experiment 2 provided an experimental context and measure likely to be more sensitive to differences in performance. Despite this, no effects of fitness relevance or of sex were found. In addition, the sex difference in overall performance efficiency (number of choices required to complete trials) found in experiment 1 was not found in experiment 2 .

\section{Experiment 3}

Experiment 3, like experiment 2, was intended to change the strong tendency to favor choices consistent with the moving target found in experiment 1 , thereby providing a more sensitive measure of the relative tendency to hunt vs. gather and, therefore, a better opportunity to reveal effects of the variables studied in these experiments. But in this case, the strategy was to modify the contingencies related to the stationary targets to make completion of trails by choosing the stationary targets relatively more attractive, rather than to increase the difficulty of choosing the moving target. In experiment 3 , the moving target behaved just as the moving target did in experiment 1 . However, the stationary targets were changed in two ways. First, there were only six stationary targets, arranged in a 2 (tall) $\times 3$ (wide) matrix. Second, following the first choice of a stationary target, the stimuli that identified them (vegetables or shape) remained visible until the end of the trial.

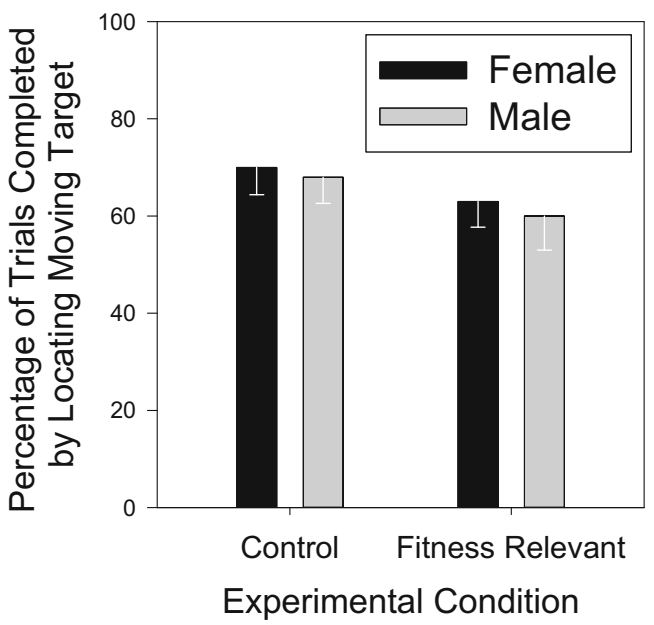

Fig. 8 The mean percentage of trials completed by choice of the moving target in experiment 2, as a function of experimental condition and sex. Error bars indicate one S.E.M. 

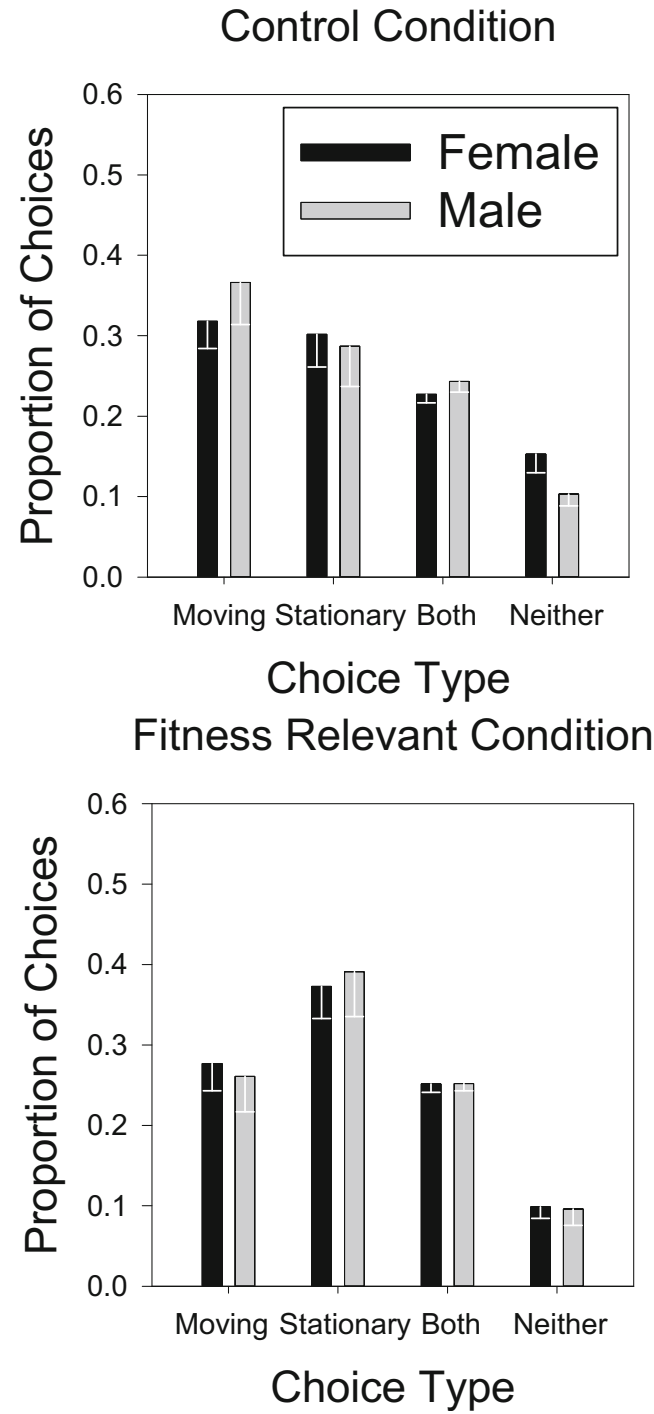

Fig. 9 The mean distributions of choices in experiment 2 consistent with the moving target, of a stationary target, both consistent with the moving target and of a stationary target, and neither consistent with the moving target nor of a stationary target. The first choice of each trial was excluded from these distributions (because no information about the moving target was available at that point). Separate distributions are shown as a function of sex and experimental condition (top vs. bottom panels). Error bars indicate one S.E.M.

The latter change removes one of at least two object location memory requirements from the gathering component of the experimental task. In experiments 1 and 2 (as well as experiment 4 below), participants needed to remember the location of stationary targets not yet chosen in order to choose them efficiently. In the present experiment, this was not required because the objects in the stationary targets were visible from the beginning of the trials until they were chosen. To choose the stationary targets efficiently, however, participants were required to remember the locations of stationary targets that had already been chosen (because they all remained visible until the end of the trial).

\section{Method}

Participants One hundred fifty-eight Villanova University students (88 female and 70 male) participated as part of a research requirement in an introductory psychology course or to receive extra-credit in a higher level course.

Materials and Apparatus The materials and apparatus used were the same as in the earlier experiments, except that the instructional sets read by participants before beginning the task were changed slightly to accommodate the changes in the task (they are provided in Appendix 2).

Procedure Participants were randomly assigned to two experimental conditions, with constraints intended to result in approximately equal numbers of female and male participants in the fitness-relevant and control conditions. There were 44 and 44 females assigned to the control and fitness-relevant conditions, respectively. The corresponding numbers for males were 33 and 37.

The procedure was identical to that of experiment 1 , with two exceptions. First, there were six stationary targets, arranged in a 2 (tall) $\times 3$ (wide) matrix of tiles. On each trial, the location of this matrix was selected randomly from among the 20 possibilities. Second, the stimuli distinguishing the stationary targets (vegetables or shape) appeared following the first choice of a stationary target (as in the earlier experiments), but they remained in place for the remainder of the trial (unlike in the earlier experiments). It should be noted that when the moving target was scheduled for the same tile as a stationary target, the moving target stimulus appeared for $3 \mathrm{~s}$ following a participant choice and then reverted back to the stationary target stimulus.

\section{Results}

The data were analyzed in the same manner as the data from experiment 2. Figure 10 shows the mean number of choices required to complete a trial as function of participant sex and experimental condition. These results were evaluated with a 2 (sex) $\times 2$ (condition) ANOVA. There was a significant effect of sex, $F(1,154)=7.42, p<0.01$, indicating that males required fewer choices than females to complete trials. There was no effect of condition, $F(1,154)<1$, and no sex $\times$ condition interaction, $F(1,154)<1$.

Figure 11 shows the percentage of trials completed by choice of the moving target, as a function of sex and condition. These results were evaluated with a $2(\operatorname{sex}) \times 2$ (condition) ANOVA. There is no evidence of effects of sex, $F(1,154)=1.29$ or condition, $F(1,154)=1.25$. There was also no significant sex $\times$ condition interaction although that factor did approach significance, $F(1,154)=$ $3.50, p=0.06$. 


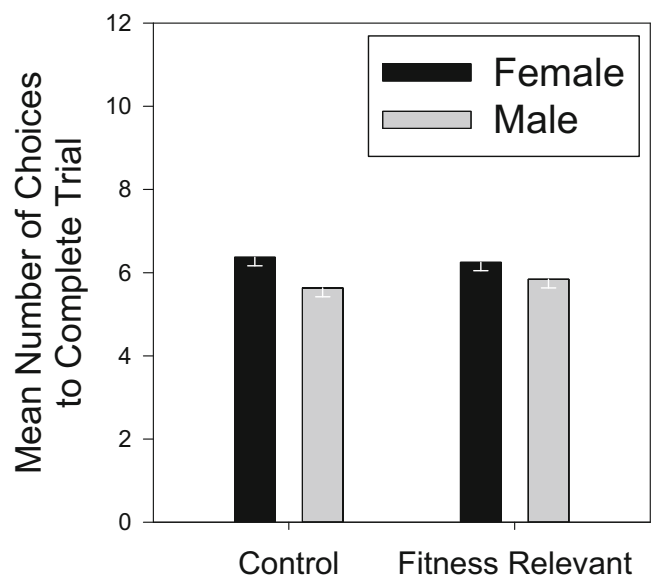

Experimental Condition

Fig. 10 The mean number of choices made to complete trials in experiment 3, as a function of experimental condition and sex. Error bars indicate one S.E.M.

Figure 12 shows distributions of choices, classified according to whether they are consistent with choice of the moving target, stationary target, both, or neither. The distributions are shown as a function of sex and experimental condition. These data were evaluated as for experiment 2 , using a 2 (choice type: consistent with moving target vs. choice of stationary target) $\times 2$ (sex $) \times 2$ (condition) ANOVA. There a significant effect of choice type, $F(1,154)=4.39$, $p<0.05$, indicating that more choices were made consistent with the moving target than of a stationary target. There was a significant interaction between choice type and condition, $F(1,154)=8.40, p<0.01$, which indicates that more choices consistent with the moving target were made in the control condition than in the fitness-relevant condition. There were no other significant effects.

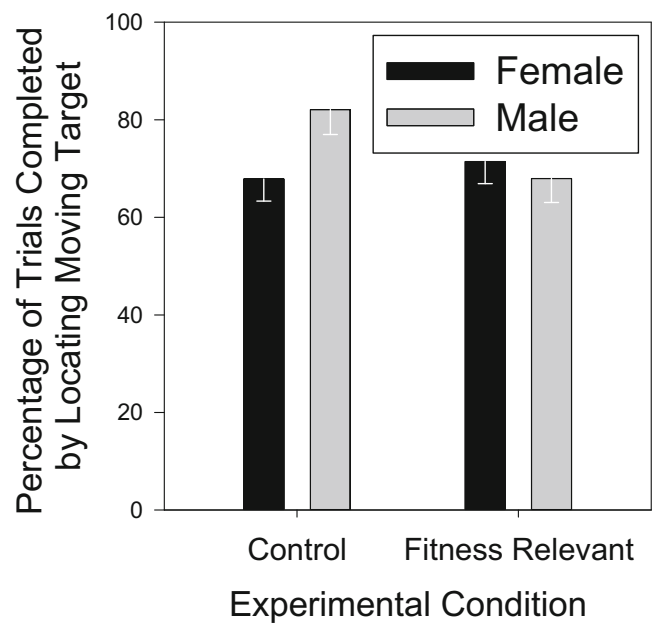

Fig. 11 The mean percentage of trials completed by choice of the moving target in experiment 3 , as a function of experimental condition and sex. Error bars indicate one S.E.M.

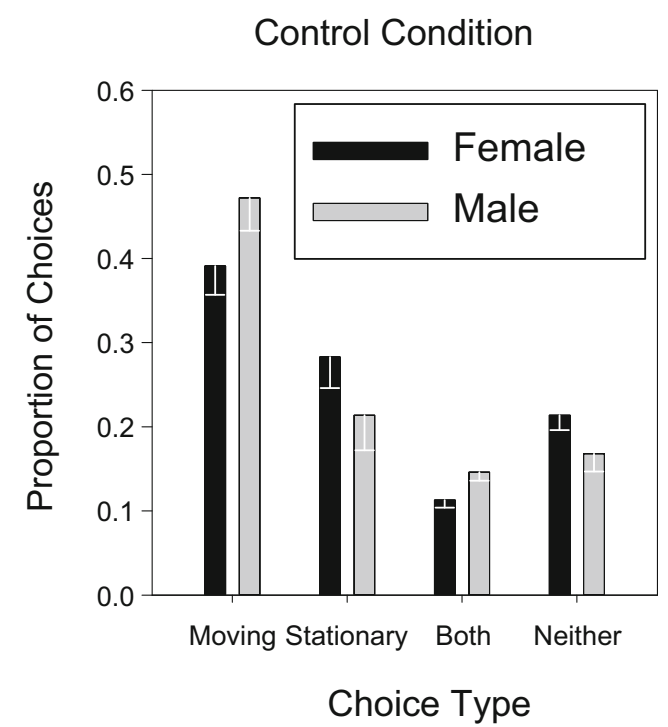

Fitness Relevant Condition

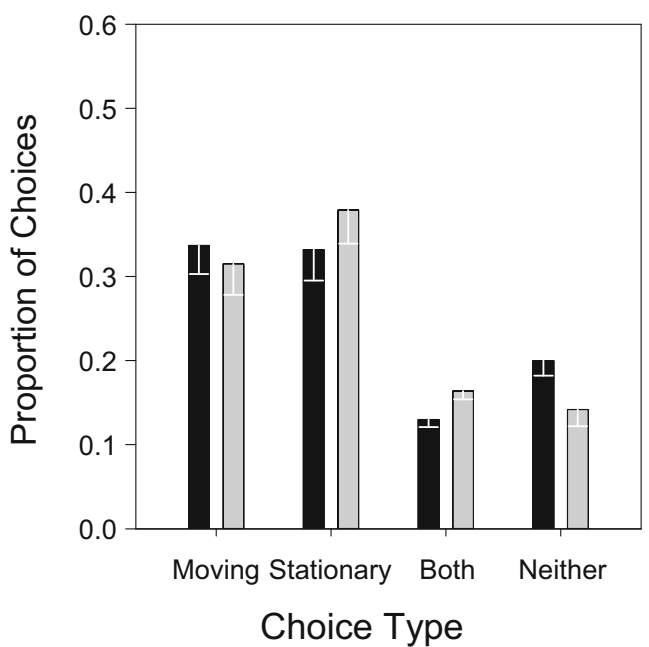

Fig. 12 The mean distributions of choices in experiment 3 consistent with the moving target, of a stationary target, both consistent with the moving target and of a stationary target, and neither consistent with the moving target nor of a stationary target. The first choice of each trial was excluded from these distributions (because no information about the moving target was available at that point). Separate distributions are shown as a function of sex and experimental condition (top vs. bottom panels). Error bars indicate one S.E.M.

\section{Discussion}

As in experiment 2, the proportions of trials completed by choice of the moving target and by choice of all the stationary targets were not strongly biased in either direction in this experiment, providing a context that should allow the effects of modulating variables to be detected more readily. Furthermore, this experiment included a relatively large number of participants to maximize the sensitivity of the experiment to any sex differences of effects of the experimental manipulation. As in experiment 1 (but not experiment 2), there 
was an effect of sex on the number of choices required to complete trials, so the task seems to have involved processes for which sex matters.

Nevertheless, there was no evidence for an effect of sex on the tendency to make choices relevant to the moving vs. stationary targets nor was there any evidence for an effect of the fitness relevance manipulation. It should be noted that the interaction between experimental condition and sex in the analysis of the tendency to complete trials by choice of the moving target did approach statistical significance. However, the form of that apparent interaction is in opposition to that predicted (female participants appear to be relatively more likely to complete trials by choice of the moving target in the fitnessrelevant condition than in the control condition).

\section{Experiment 4}

Experiment 4 was intended to explore somewhat different conditions that might reveal a sex difference in choices of stationary vs. moving targets. In all of the previous experiments, the stationary targets were arranged in a structured spatial configuration (i.e., a $3 \times 3$ or $3 \times 2$ matrix). In the present experiment, the locations of the stationary targets were selected randomly from the 36 tile locations and so they had no consistent spatial configuration. There were six stationary targets on each trial.

To include a memory requirement in the task of choosing the stationary targets, we used a technique that was a compromise between revealing the stationary targets only briefly following the first choice of a stationary target (experiments 1 and 2) and revealing the stationary targets constantly until each was chosen (experiment 3). Specifically, the stationary targets were revealed by the (vegetable image) stimulus at the beginning of each trial. The tiles in the stationary targets reverted back to the background image following the first choice of any tile and they remained hidden until they were chosen (at which point the background image was replaced by the vegetable image until the end of the trial). Thus, to choose stationary targets effectively, participants had to study the location of the stationary targets before making their first choice and then remember those locations during the trial. However, they were not required to remember the locations of stationary targets that had already been chosen during a trial, as those locations were visible. Thus, this experiment included memory requirements for the gathering component of the task that were the complement of the memory requirements in experiment 3 .

The goal of this experiment was focused on the possibility of a sex difference in choices of moving vs. stationary targets and so we chose to test all participants in the fitness-relevant conditions of the earlier experiments.

\section{Method}

Participants One hundred twenty-one Villanova University students (66 female and 55 male) participated as part of a research requirement in an introductory psychology course.

Materials and Apparatus The materials and apparatus used were the same as in the earlier experiments, except that the instructional sets read by participants before beginning the task were changed slightly to accommodate the changes in the task (they are provided in Appendix 2).

Procedure The procedure was identical to that of experiment 3 with the following exceptions. First, all participants were tested under the fitness-relevant conditions of the earlier experiments. They were assigned randomly to one of the three moving targets (animals) and one of the three stationary targets (vegetables). Second, the six stationary targets were selected for each trial randomly (without replacement) from among the 36 tile locations. Finally, the locations of the stationary targets were revealed (vegetable images present in the target locations) when the participant began the trial by clicking the "Begin Trial" button. They remained visible until the first choice of any location, following which they reverted to the background image. When a stationary target was chosen, it was "marked" by the presence of the vegetable image until the end of the trial.

\section{Results}

The data were analyzed in the same manner as the data from experiments 2 and 3, except that experimental condition was not a factor. Figure 13 shows the mean number of choices required to complete a trial for female and male participants. The values did not differ, $F(1,119)<1$. Figure 14 shows the percentage of trials completed by choice of the moving target for females and male participants. These values did not differ, $F(1,119)<1$. Figure 15 shows distributions of choices for female and male participants, classified according to whether they are consistent with choice of the moving target, stationary target, both, or neither. These data were evaluated as for experiments 2 and 3 , using a 2 (choice type: consistent with moving target vs. choice of stationary target $) \times 2$ (sex) ANOVA. There was a significant effect of choice type, $F(1,119)=54.47, p<0.001$, indicating that more choices were made of stationary targets than to tiles 


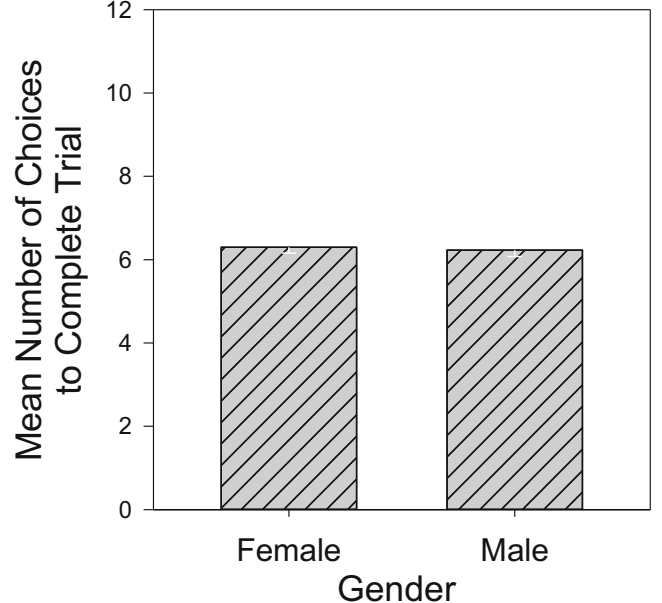

Fig. 13 The mean number of choices made by female and male participants to complete trials in experiment 4. Error bars indicate one S.E.M.

consistent with the moving target. There was not a significant effect of sex, $F(1,119)=2.66$ nor (more importantly) an interaction between choice type and sex, $F(1,119)<1$.

\section{Discussion}

The procedure of this experiment encouraged relatively more choices of stationary targets than were found in the earlier experiments. Thus, this experiment increases the range of conditions under which sex differences in choices of the two kinds of targets could be revealed. Again, however, there was no indication of sex differences in the tendency to make choices consistent with finding the moving target as opposed to choosing all of the stationary targets.

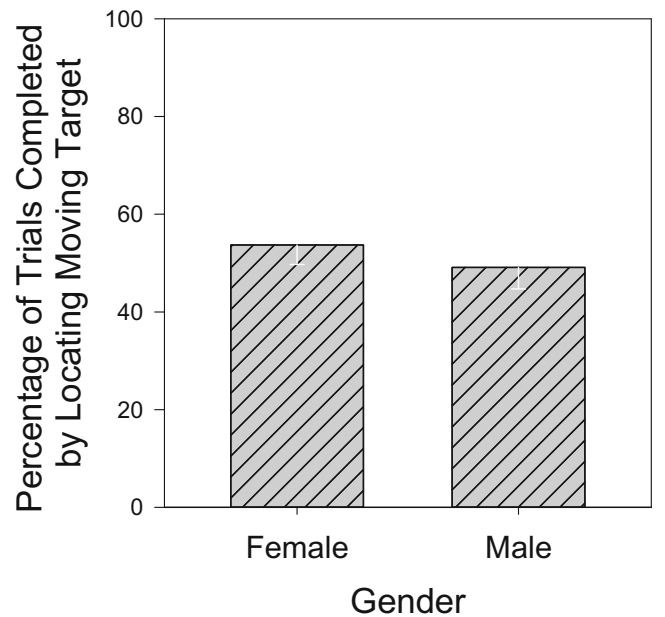

Fig. 14 The mean percentage of trials completed by choice of the moving target by female and male participants in experiment 4. Error bars indicate one S.E.M.

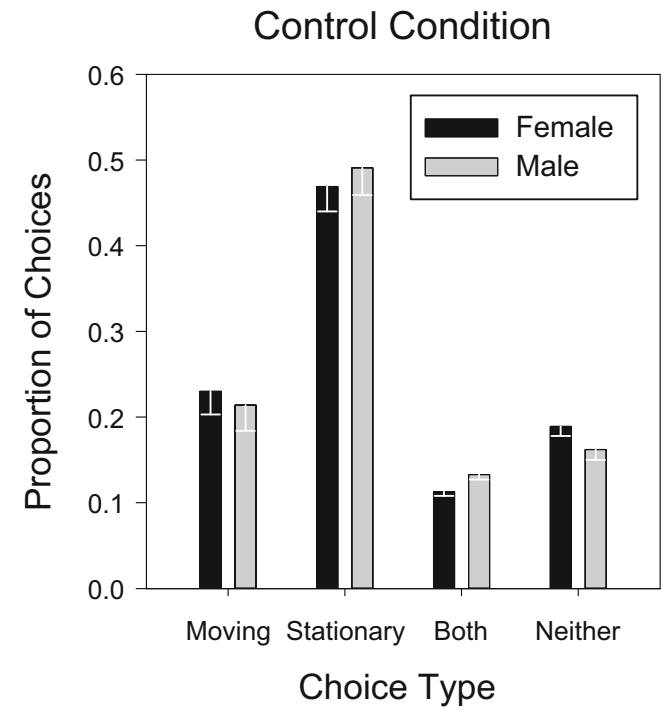

Fig. 15 The mean distributions of choices in experiment 4 consistent with the moving target, of a stationary target, both consistent with the moving target and of a stationary target, and neither consistent with the moving target nor of a stationary target. The first choice of each trial was excluded from these distributions (because no information about the moving target was available at that point). Separate distributions are shown for female and male participants. Error bars indicate one S.E.M.

\section{General Discussion}

The results of three experiments in which fitness relevance was manipulated provide no evidence for an effect of fitness relevance on spatial performance in this task. No differences in choice efficiency (the number of choices required to complete trials) were found. This was the case regardless of whether the task encouraged mostly choices of moving targets (experiment 1 ) or relatively more choices of stationary targets (experiments 2 and 3 ).

It is difficult to know why an effect of fitness relevance was not found. It might be that the fitness-relevant condition did not sufficiently evoke "survival processing" (Nairne 2010). However, the manipulation included instructional sets that were very similar to those used in the Nairne studies, as well as additional support for survival processing from the images used. A second possibility is that the control condition included sufficient elements of spatial processing related to hunting and gathering that "survival processing" occurred in both conditions. Alternatively, it may be that survival processing affects only a subset of cognitive processes, even among those processes that were central to important human behaviors during large portions of human evolutionary history and that the spatial performance involved in the present task is not among them. The survival processing that is effective in the Nairne task is nominally a matter of memory encoding. The effect of survival-relevant processing may be restricted to memory encoding or even to encoding necessary for modulation of incidental recall of verbal material. It may not modulate spatial 
cognition or, at least, not the spatial cognition involved in the present task. Another possibility is that the kinds of processes that constitute effective survival processing are evoked to different extents by different kinds of tasks. The present results do not favor any of these possibilities over the others, but we hope that these results help point the way toward successful explorations of them.

The results of all four experiments failed to reveal sex differences in the tendency to make choices consistent with the moving target ("hunting") vs. remembering and choosing the stationary targets ("gathering"). Again, the explanation for this null result is not clear. It should be noted that reports from the survival processing paradigm do not generally include performance reported as a function of sex. An exception (Nairne et al. 2009) reports no evidence for sex differences in recall of items in the "hunting" and "gathering" scenario conditions.

Unlike earlier experiments inspired by Silverman and Eals's work, the requirements of the present task and the measures of performance used in these experiments were not intended to isolate object location memory. The gathering task did include two forms of object location memory (memory for the locations of stationary targets not yet chosen in experiments 1,2, and 4; memory for the locations of stationary targets chosen earlier during the trial in experiments 1,2 , and 3). However, we measured the relative tendency to locate a single moving target (hunt) vs. locate multiple stationary targets (gather). This tendency would presumably be influenced by object location memory, but would also reflect many other abilities and tendencies. The intention was to use a task that could be completed in either of these two ways and measure both the efficiency of task completion and the relative tendency to complete the task in the two ways, thereby allowing any and all of the skills, abilities, and tendencies related to locating a single moving target vs locating multiple stationary targets to play a role in the results. Despite this strategy of allowing a large range of factors that might produce a sexual differential of hunting and gathering tendencies, none was found.

One possible interpretation of the failure to find sex differences in the present experiments is that, in fact, the essential components of hunting and gathering were not present in our desktop model task. There are certainly many features of real world hunting and gathering that are missing from our task and so there are many candidate critical features that might need to be present to produce a sex difference in the relative tendency to hunt or gather in females and males. However, it may be worth pointing out that a desktop tasks similar to the ones used in these experiments has been used to explore spatial pattern learning and has been shown to produce patterns of results that are very similar to spatial pattern learning found in full-scale spatial tasks involving walking among locations in a two-dimensional matrix (e.g., Nowak et al. 2015; Sturz et al. 2009; Sturz et al. 2010). Thus, at least some aspects of real world search and choice among spatial locations are preserved in tasks like the one used in these experiments.

We hope that the lack of evidence for a sex difference in performance on this task, as well as for an effect of fitness relevance, will be useful in guiding future research on the relationships among hunting-related processes, gatheringrelated processes, sex differences in spatial cognition, and human evolutionary history. These results may also serve as a cautionary note regarding evidence in evolutionary psychology (or any field). Consideration of evolutionary pressures on cognitive processes make many interesting and potentially important predictions, but many (almost certainly including many that have been empirically tested but are not published; Rosenthal 1979) are not supported empirically for reasons that are difficult to discern.

Acknowledgments Experiment 1 formed the basis of an M.S. thesis by Jared Batterman. This research was approved by the Institutional Review Board at Villanova University.

\section{Appendix 1: Video Samples of Trials from Experiments 1 and 4}

Six examples of trials using the procedure of experiments 1 (examples 1-6) and 4 (examples 7 and 8). These were completed for demonstration purposes (they are not trials completed by participants).

\section{Example Trials from Experiment 1}

Example 1-fitness-relevant stimuli. "Hunting" choices made. Trial completed by choice of moving target ("hunting"). https://youtu.be/EUhhVrf4rtk

Example 2-fitness-relevant stimuli. "Gathering" choices made after first stationary target located. Trial completed by choice of stationary targets ("gathering"). https://youtu.be/9m3lbq0bdEI

Example 3-fitness-relevant stimuli. Both "gathering" choices and "hunting" choices made. Trial completed by choice of stationary targets ("gathering"). https:// youtu.be/2RHMBb60_sk

Example 4-control stimuli. "Hunting" choices made. Trial completed by choice of moving target ("hunting"). https://youtu.be/zE8QrHB9Egs

Example 5-control stimuli. "Gathering" choices made after first stationary target located. Trial completed by choice of stationary targets ("gathering"). https://youtu. be/dM8FSei2sgk

Example 6-control stimuli. Both "gathering" choices and "hunting" choices made. Trial completed by choice of moving target ("hunting"). https://youtu.be/ DFgYLGeaybI 


\section{Example Trials from Experiment 4}

Example 7-trial completed by choice of moving target ("hunting"). https://youtu.be/7iuiHHTJ4dE

Example 8-trial completed by choice of stationary targets ("gathering"). https://youtu.be/DtrYf81dxL0

\section{Appendix 2: Participant Instructions for Experiments 3 and 4}

The participant instructions for experiment 1 appear in the text. The participant instructions for experiment 2 were identical to those for experiment 1 .

\section{Participant Instructions for Experiment 3}

Fitness-Relevant Condition

We would like you to imagine that you are living long ago in the grasslands of a foreign land. As a part of a small group, you are in charge of contributing food for your tribe.

You can either hunt game or gather edible fruits, nuts, or vegetables. Hunters often have to travel great distances in order to find food, and once they've found it, they often have to track it before they are able to capture it. Gatherers often have to have knowledge about the locations and seasonal availability of edible foods.

When you have finished reading these instructions, you will be presented with a matrix of grassland locations. To begin securing food, simply click any of the locations, following which you will see an animal appear for a short time before once again disappearing. The location in which the animal appeared was its location at the time you clicked. To catch it, thus securing meat for your tribe, you will need to click the location in which you anticipate the animal will be next. You will also see a gathering area (a fertile patch of land, containing fruits, nuts, and/or vegetables). If you choose a location with fruit, nuts or vegetables, it will become empty to indicate that you have gathered the food item. To gather enough food for your tribe, you will need to click all the locations inside the gathering area. You will be sent out on 40 expeditions to secure food, each of which can be completed by hunting or gathering. Either strategy can allow you to get enough food to feed your tribe. However, time is of the essence, and it is your goal to finish each expedition as quickly and as accurately as you can. This will also allow you to complete the experiment more quickly.

\section{Control Condition}

We would like you to complete a series of computer games. Your job is to finish them as quickly and as accurately as possible. Each game can be completed either by clicking a single moving shape, or by clicking several locations which contain a hidden stationary shape.

When you have finished reading these instructions, you will be presented with a matrix consisting of black tiles. To begin a game, simply click any of the tiles, following which you will see the mobile shape appear for a short time before once again disappearing. The location in which it appeared was its location at the time you clicked. To catch it, you will need to click the tile that you anticipate it will be next. You will also see an area containing stationary shapes. If you click a stationary shape, it will disappear to indicate that you have chosen it. Choosing all of the stationary shapes will end the game. You will complete 40 games, each of which can be completed by finding and clicking the single moving shape or by finding all of the hidden stationary shapes. Either strategy will allow you to complete each game, thus bringing you one step closer to the end of the experiment. However, time is of the essence, and it is your goal to find the shapes as quickly and as accurately as you can.

\section{Participant Instructions for Experiment 4}

\section{Fitness-Relevant Condition}

We would like you to imagine that you are living long ago in the grasslands of a foreign land. As a part of a small group, you are responsible to contribute food for your extended family and tribe.

You can either hunt game or gather edible fruits, nuts, or vegetables. Hunters often have to travel great distances in order to find food, and once they've found it, they often have to track it before they are able to capture it. Gatherers often must remember the locations of edible foods.

When you have finished reading these instructions, you will be presented with a matrix of grassland locations. 
To begin securing food, simply click any of the locations, following which you will see an animal appear for a short time before once again disappearing. The location in which the animal appeared was its location at the time you clicked. To catch it, thus securing meat for your tribe, you will need to click the location in which you anticipate the animal will be next. You will also see gathering locations (containing fruits, nuts, and/or vegetables). After you make a choice, the gathering locations will no longer be visible. But if you remember where they were, you will be able to gather the hidden items that are still there. After you choose a location with fruit, nuts or vegetables, it will become visible again to indicate that you have already gathered the food item. To gather enough food for your tribe, you will need to click all five locations containing a fruit, nut, or vegetable food item. You will be sent out on 40 expeditions to secure food, each of which can be completed by either hunting or gathering. Either strategy can allow you to get enough food to feed your tribe. However, time is of the essence, and it is your goal to finish each expedition as quickly and as accurately as you can. This will also allow you to complete the experiment more quickly.

\section{Control Condition}

We would like you to complete a series of computer games. Your job is to finish them as quickly and as accurately as possible. Each game can be completed either by clicking a single moving shape, or by clicking several locations which contain a hidden stationary shape.

When you have finished reading these instructions, you will be presented with a matrix consisting of black tiles. To begin a game, simply click any of the tiles, following which you will see the mobile shape appear for a short time before once again disappearing. The location in which it appeared was its location at the time you clicked. To catch it, you will need to click the tile that you anticipate it will be next. You will also see locations containing stationary shapes. After you make a choice, the locations containing stationary shapes will no longer be visible. But if you remember where they were you will be able to choose the hidden shapes that are still there. After you choose a location with a stationary shape, the shape will become visible again to indicate that you have already chosen it. Choosing all of the stationary shapes will end the game. You will complete 40 games, each of which can be completed by finding and clicking the single moving shape or by finding all of the hidden stationary shapes. Either strategy will allow you to complete each game, thus bringing you one step closer to the end of the experiment. However, time is of the essence, and it is your goal to find the shapes as quickly and as accurately as you can.

\section{References}

Barnfield, A. M. C. (1999). Development of sex differences in spatial memory. Perceptual and Motor Skills, 89, 339-350.

Cashdan, E., Marlowe, F. W., Crittenden, A., Porter, C., \& Wood, B. M. (2012). Sex differences in spatial cognition among Hadza foragers. Evolution and Human Behavior, 33, 274-284. doi:10.1016/j. evolhumbehav.2011.10.005.

Choi, J., \& L'Hirondelle, N. (2005). Object location memory: a direct test of the verbal memory hypothesis. Learning and Individual Differences, 15, 237-245. doi:10.1016/j.lindif.2005.02.001.

Choi, J., \& Silverman, I. (1996). Sexual dimorphism in spatial behaviors: applications to route learning. Evolution and Cognition, 2, 165-171.

Dabbs, J. M., Chang, E. L., Strong, R. A., \& Milun, R. (1998). Spatial ability, navigation strategy, and geographic knowledge among men and women. Evolution and Human Behavior, 19, 89-98.

Eals, M., \& Silverman, I. (1994). The hunter-gatherer theory of spatial sex differences: proximate factors mediating the female advantage in recall of object arrays. Ethology and Sociobiology, 15, 95-105. doi:10.1016/0162-3095(94)90020-5.

Ecuyer-Dab, I., \& Robert, M. (2004). Have sex differences in spatial ability evolved from male competition for mating and female concern for survival? Cognition, 91, 221-257. doi:10.1016/j.cognition. 2003.09.007.

Gaulin, S. J. C. (2006). Evolutionary psychology. In J. H. Kaas (Ed.), Evolution of nervous systems (Vol. 4, pp. 497-502). Oxford: Elsevier.

Gaulin, S. J. C., \& FitzGerald, R. W. (1986). Sex differences in spatial ability: an evolutionary hypothesis and test. The American Naturalist, 127, 74-88.

Gaulin, S. J. C., \& Hoffman, H. A. (1998). Evolution and development of sex differences in spatial ability. In L. Betzig, M. B. Mulder, \& P. Turke (Eds.), Human reproductive behavior: a Darwinian perspective (pp. 129-152). Cambridge: Cambridge University Press.

Halpern, D. F. (2000). Sex differences in cognitive abilities. Mahwah: Lawrence Erlbaum Associates.

Honda, A., \& Nihei, Y. (2009). Sex differences in object location memory: the female advantage of immediate detection of changes. Learning and Individual Differences, 19, 234-237. doi:10.1016/j. lindif.2008.11.005.

Iachini, T., Sergi, I., Ruggiero, G., \& Gnisci, A. (2005). Gender differences in object location memory in a real three-dimensional environment. Brain and Cognition, 59, 52-59. doi:10.1016/j.bandc. 2005.04.004.

James, T. W., \& Kimura, D. (1997). Sex differences in remembering the locations of objects in an array: location-shifts versus location-exchanges. Evolution and Human Behavior, 18, 155-163.

Kang, S. H. K., McDermott, K. B., \& Cohen, S. M. (2008). The mnemonic advantage of processing fitness-relevant information. Memory \& Cognition, 36, 1151-1156. doi:10.3758/MC.36.6.1151.

Leibowitz, B. K., \& Brown, M. F. (1999). Sex differences in spatial search and pattern learning in the rat. Psychobiology, 27, 364-371. 
Linn, M. C., \& Petersen, A. C. (1985). Emergence and characterization of sex differences in spatial ability: a meta-analysis. Child Development, 56, 1479-1498.

McBurney, D. H., Gaulin, S. J. C., Devineni, T., \& Adams, C. (1997). Superior spatial memory of women: stronger evidence for the gathering hypothesis. Evolution and Human Behavior, 183, 165-174. doi:10.1016/S1090-5138(97)00001-9.

Nairne, J. S. (2010). Adaptive memory: evolutionary constraints on remembering. In B. H. Ross (Ed.), The psychology of learning and motivation: advances in research and behavior (Vol. 53, pp. 1-32). San Diego: Academic. doi:10.1016/S0079-7421(10)53001-9.

Nairne, J. S., \& Pandeirada, J. N. S. (2008). Adaptive memory: remembering with a stone-age brain. Current Directions in Psychological Science, 17, 239-243.

Nairne, J. S., \& Pandeirada, J. N. S. (2010). Adaptive memory: ancestral priorities and the mnemonic value of survival processing. Cognitive Psychology, 61, 1-22. doi:10.1016/j.cogpsych.2010.01.005.

Nairne, J. S., Thompson, S. R., \& Pandeirada, J. N. S. (2007). Adaptive memory: survival processing enhances retention. Journal of Experimental Psychology. Learning, Memory, and Cognition, 33, 263-273. doi:10.1037/0278-7393.33.2.263.

Nairne, J. S., Pandeirada, J. N. S., Gregory, K. J., \& VanArsdall, J. E. (2009). Adaptive memory: fitness-relevance and the hunter-gatherer mind. Psychological Science, 20, 740-746.

Nairne, J. S., VanArsdall, J. E., Pandeirada, J. N. S., \& Blunt, J. R. (2012). Adaptive memory: enhance location memory after survival processing. Journal of Experimental Psychology. Learning, Memory and Cognition, 38, 495-501. doi:10.1037/a0025728.

Nowak, N. T., Murali, A., \& Driscoll, I. (2015). Factors related to sex differences in navigating a computerized maze. Journal of Environmental Psychology, 43, 136-144. doi:10.1016/j.jenvp. 2015.06.007.

Otgaar, H., Smeets, T., \& van Bergen, S. (2010). Picturing survival memories: enhanced memory after fitness-relevant processing occurs for verbal and visual stimuli. Memory \& Cognition, 38, 23-28. doi:10. 3758/MC.38.1.23.

Postma, A., Izendoorn, R., \& De Haan, E. H. F. (1998). Sex differences in object location memory. Brain and Cognition, 36, 334-345. doi:10. 1006/brcg.1997.0974.
Postma, A., Jager, G., Kessels, R. P. C., Koppeschaar, H. P. F., \& van Honk, J. (2004). Sex differences for selective forms of spatial memory. Brain and Cognition, 54, 24-34. doi:10.1016/S0278-2626(03) 00238-0.

Rosenthal, R. (1979). The file drawer problem and tolerance for null results. Psychological Bulletin, 86, 638-641. doi:10.1037/00332909.86.3.638

Saucier, D. M., Schultz, S. R., Keller, A. J., Cook, C. M., \& Binsted, G. (2007). Sex differences in object location memory and spatial navigation in Long-Evans rats. Animal Cognition, 11, 129-137.

Silverman, I., \& Eals, M. (1992). Sex differences in spatial abilities: evolutionary theory and data. In J. H. Barkow, L. Cosmides, \& J. Tooby (Eds.), The adapted mind: evolutionary psychology and the generation of culture (pp. 531-549). New York: Oxford Press.

Silverman, I., Choi, J., \& Peters, M. (2007). The hunter-gatherer theory of sex differences in spatial abilities: data from 40 countries. Archives of Sexual Behavior, 36, 261-268. doi:10.1007/s10508-006-9168-6.

Sturz, B. R., Brown, M. F., \& Kelly, D. M. (2009). Facilitation of learning spatial relations among locations by visual cues: implications for theoretical accounts of spatial learning. Psychonomic Bulletin and Review, 16, 306-312. doi:10.3758/PBR.16.2.306.

Sturz, B. R., Kelly, D. M., \& Brown, M. F. (2010). Facilitation of learning spatial relations among locations by visual cues: generality across spatial configurations. Animal Cognition, 13, 341-349. doi:10. 1007/s10071-009-0283-3.

Vashro, L., \& Cashdan, E. (2015). Spatial cognition, mobility, and reproductive success in northwestern Namibia. Evolution and Human Behavior, 36, 123-129. doi:10.1016/j.evolhumbehav.2014.09.009.

Voyer, D., Voyer, S., \& Bryden, M. P. (1995). Magnitude of sex differences in spatial abilities: a meta-analysis and consideration of critical variables. Psychological Bulletin, 117, 250-270. doi:10.1037/00332909.117.2.250.

Voyer, D., Postma, A., Brake, B., \& Imperiato-McGinley, J. (2007). Gender differences in object location memory: a meta-analysis. Psychonomic Bulletin \& Review, 14, 23-38.

Weinstein, Y., Bugg, J. M., \& Roediger, H. L. (2008). Can the survival recall advantage be explained by basic memory processes? Memory \& Cognition, 36, 913-919. doi:10.3758/MC.36.5.913. 